\title{
Anxiolytic-like effect of trans-2-decenoic acid ethyl ester in stress-induced anxiety-like model mice
}

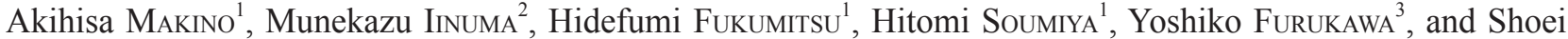 \\ FURUKAWA ${ }^{1}$ \\ ${ }^{1}$ Laboratory of Molecular Biology, Department of Biofunctional Analysis, Gifu Pharmaceutical University, Daigaku-nishi 1-25-4, Gifu \\ 501-1196, Japan; ${ }^{2}$ Laboratory of Pharmacognosy, Gifu Pharmaceutical University, Daigaku-nishi 1-25-4, Gifu 501-1196, Japan; and \\ ${ }^{3}$ Department of Pharmaceutical Pharmacology, Faculty of Pharmaceutical Sciences, Matsuyama University, Bunkyo-cho 4-2, Matsuyama, \\ Ehime 790-8578, Japan
}

(Received 9 August 2013; and accepted 26 August 2013)

\begin{abstract}
Brain-derived neurotrophic factor (BDNF) is considered to be involved in the etiology and treatment of stress-related mood disorders including anxiety. However, application of BDNF itself has many technical and pharmacological problems such as instability and/or a lack of appropriate delivery systems. To overcome these, we developed trans-2-decenoic acid ethyl ester (DAEE) as a stable and small molecule with BDNF-like activities. In the present study, we tested the activities of DAEE on a stress-induced anxiety-like mouse model. Mice were kept in cages and subjected to 3 sets of sequential leaning, drenching, and rotation as chronic mild stresses applied for 1-2 days over a 3-week period; and the anxiety-like symptom (reduced time spent in open arm of the maze) was evaluated by use of the elevated plus-maze test. A daily intraperitoneal administration of DAEE competed against the expression of the anxiety-like symptom when administered during the stress-loading period, and became therapeutic when administered after the stress-loading. This activity was accompanied by amelioration of the stress-induced reduction in the levels of BDNF and neurotrophin-3 mRNAs and phosphorylated extracellular signal-regulated kinases (ERK) $1 / 2$ in the hippocampus. These results demonstrated that DAEE behaved like an anxiolytic and ameliorated this characteristic anxiety-like symptom, suggesting that DAEE may be a promising candidate for a novel anxiolytic with a new mechanism of action.
\end{abstract}

Brain-derived neurotrophic factor (BDNF), a member of neurotrophin neurotrophic factor family, plays roles in the maintenance of neuronal function and plasticity during development and adulthood $(7,27)$. BDNF is highly expressed in the hippocampus, and a growing body of evidence indicates that hippocampal BDNF is involved in the etiology and treatment of stress-related mood disorders including

Address correspondence to: Dr. Shoei Furukawa Laboratory of Molecular Biology, Department of Biofunctional Analysis, Gifu Pharmaceutical University, Daigaku-nishi 1-25-4, Gifu 501-1196, Japan

Tel: +81-58-230-8100, Fax: +81-58-230-8105

E-mail: furukawa@gifu-pu.ac.jp depression and anxiety $(11,25)$. Exposure to stressful events is a primary cause of the development of mood disorders, and the hippocampus is particularly sensitive to stress. For example, exposure to a stressor inhibits neurogenesis in the dentate gyrus $(13,14)$, and causes a reduction in hippocampal volume $(24,30)$. Importantly, such exposure also reduces the level of BDNF in the hippocampus (32). These findings suggest that a reduction in neurotrophic support by BDNF causes stress-induced hippocampal damage $(10,11)$. Therefore, some endogenous and exogenous compounds such as antidepressants (21), histone deacetylase inhibitors (26), BDNF (23), and 7,8-dihydroxyflavone (DHF), which is a TrkB agonist $(18,19)$, modulate or may modu- 
late depression- and/or anxiety-like symptoms in both humans and rodents, either directly or indirectly, via BDNF/TrkB signaling mechanisms.

Earlier we found that medium-chain fatty acids with 8-12 carbons and their esters facilitate the phosphorylation of mitogen-activated protein kinases (MAPK)/extracellular signal-regulated kinases (ERK) $1 / 2$ in cultured embryonic cortical/hippocampal neurons (15). In particular, trans-2-decenoic acid ethyl ester (DAEE; see Fig. 1) has the most potent activity among the compounds tested $(15,29)$. DAEE also activates phosphatidylinositol 3-kinase and cAMP-response element-binding protein (CREB), and increases the expression of mRNAs of neurotrophins and the content of synapse-specific proteins (29), indicating that DAEE generates similar intracellular signaling as neurotrophins including BDNF. These observations prompted us to examine the efficacy of DAEE on stress-induced depression- and anxiety-like symptoms of animal models.

Earlier we confirmed the therapeutic effects of DAEE on a depression-like symptom as evidenced by extension of the immobility time in the tail suspension test (12), but had not yet examined its anxiolytic-like activity. In the present study, we estimated the anxiolytic-like effect of DAEE on chronic stressloaded mice and examined its effect on intracellular signaling and gene expression in the hippocampus. We found that DAEE behaved like an anxiolytic, ameliorating a characteristic anxiety-like symptom.

\section{MATERIALS AND METHODS}

Animals. Seven-week-old male ddY mice (Japan SLC, Hamamatsu, Japan), weighing 35-40 g, were used. The ddY strain is an outbred one, and has been maintained as a closed colony with good reproductive performance and superior growth. In Japan, this strain has been widely used in various fields of research. The mice were bred under conditions of constant temperature $\left(23 \pm 2{ }^{\circ} \mathrm{C}\right)$, humidity $(55 \pm 10 \%)$, and a 12-h light/12-h dark cycle with food and water freely available. All animal experiments were performed according to the Guideline for Care and Use of Laboratory Animals of Gifu Pharmaceutical University.

Drug treatment. DAEE (see Fig. 1), purchased from Sigma-Aldrich Corp (St. Louis, MO, USA), was dissolved in dimethylsulfoxide (DMSO) and diluted 1000 -fold in phosphate-buffered saline (PBS). It was administered intraperitoneally (i.p.) into the animals at a dose of 20,100 or $500 \mu \mathrm{g} / \mathrm{kg}$ body weight. Flu-

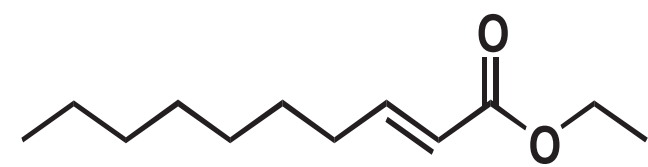

Fig. 1 Chemical structure of DAEE.

voxamine, a popularly prescribed selective serotonin reuptake inhibitor (SSRI), was obtained from Toronto Research Chemicals Inc. (Toronto, Canada), dissolved directly in PBS containing $0.1 \%$ DMSO, and administered i.p. at $1 \mathrm{mg} / \mathrm{kg}$ body weight. Fluvoxamine was used as a typical anxiolytic to suppress anxiety-like symptom. Control animals received vehicle (PBS) containing 0.1\% DMSO without DAEE. The volume of drug solution administered was $0.25 \mathrm{~mL} /$ mouse.

Stress-induced anxiety-like model mice. Chronic mild stress (CMS) was applied to the mice according to a previously published method (17) with a slight modification (Fig. 2). Briefly, the mice were exposed to CMS, which consisted of 3 different and sequential stress situations: inclining their cage by 20 degrees from the horizontal (CMS1), keeping them on chip bedding fully wetted with water (CMS2), and shaking their cages at $180 \mathrm{rpm}$ by use of a rotatory shaker (CMS3). The CMS1, CMS2, and CMS3 were sequentially applied for 48,24 , and $24 \mathrm{~h}$, respectively, with a 24 -h interval between each CMS. This set of stress-loading was repeated 3 times over a 20-day period (Fig. 2).

In order to evaluate the protective activity against the anxiety-like symptom, we administered the drug once a day for 21 days starting at the time of the first stress-loading till the end of the stress-loading period (Fig. 2A). On the other hand, for the experiments to test the therapeutic activity, the drug was injected once a day for 7 or 14 days starting the next day after the end of the stress-loading (Fig. 2B).

Elevated plus-maze test. This is a standard test to measure the fear and anxiety-like state. After treatment, the animals were placed in the center of a 4-arm-maze $(30 \times 5 \mathrm{~cm} / \mathrm{arm})$ elevated to a height of $50 \mathrm{~cm}$, in which 2 arms were open and 2 were closed (33). The number of times the animal entered each of the arms and the time spent in each arm was recorded during a 5-min test period. The procedure was conducted in a sound-attenuated room.

Reverse transcription-polymerase chain reaction. Hippocampi were dissected out from anesthetized 
A Day $0 \begin{array}{llllllllllllllllllllll} & 1 & 2 & 3 & 4 & 5 & 6 & 7 & 8 & 9 & 10 & 11 & 12 & 13 & 14 & 15 & 16 & 17 & 18 & 19 & 20 & 21\end{array}$

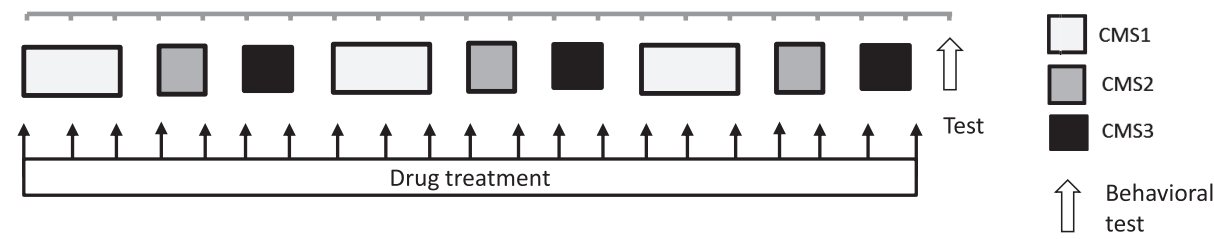

B Day $0 \begin{array}{lllllllllllllllllllll} & 1 & 2 & 3 & 4 & 5 & 6 & 7 & 8 & 9 & 10 & 11 & 12 & 13 & 14 & 15 & 16 & 17 & 18 & 19 & 20\end{array}$

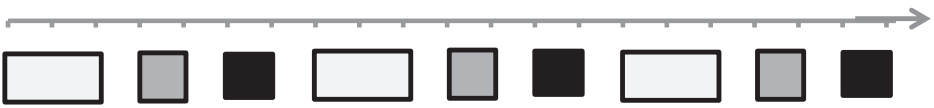

Day $\begin{array}{lllllllllllllll}21 & 22 & 23 & 24 & 25 & 26 & 27 & 28 & 29 & 30 & 31 & 32 & 33 & 34 & 35\end{array}$

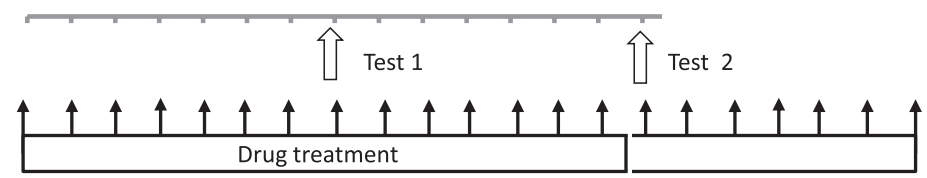

Fig. 2 Protocols for stress-loading and drug administration. A: Protocol of experiments for evaluation of protective activity. Mice were given i.p. the drug once a day for 21 days (indicated by the arrows) during exposure to the 3 types of CMSs (CMS1, CMS2 or CMS3) (indicated as boxes colored white, gray, and black, respectively). Then, the time spent in open arms and the number of entries into all arms in the elevated plus-maze test were evaluated at 1 day after the end of the stress-loading period (open arrow). B: Protocol of experiments for evaluation of therapeutic activity. Mice were subjected to the 3 different CMSs (CMS1, CMS2 or CMS3) for 20 days. The CMSs are illustrated as boxes as defined in "A." Each drug was given i.p. once a day for 7 days or 14 days starting 1 day after the end of the stress-loading period, and the time spent in open arms and the number of entries into all arms in the elevated plus-maze test were then evaluated (open arrows).

animals, and RNA was prepared by using Trizol Reagent (Invitrogen) according to the manufacturer's instructions. All RNA samples were treated with DNase I to remove contaminating genomic DNA. Semi-quantitative RT-PCR was performed as described previously (22) to assess mRNA levels of nerve growth factor (NGF), BDNF, and neurotrophin (NT)-3, with $\beta$-actin mRNA used as the internal control. The amplification was carried out with a thermal cycler at $94^{\circ} \mathrm{C}$ for $5 \mathrm{~min}$, followed by 24 38 cycles consisting of $94^{\circ} \mathrm{C}$ for $30 \mathrm{~s}, 60-65^{\circ} \mathrm{C}$ for $1 \mathrm{~min}$, and $72^{\circ} \mathrm{C}$ for $45 \mathrm{~s}$. The sequences of primers, annealing temperatures, and size of PCR products were the following: $\beta$-actin, 5 '-GTGGGCCGCTCTA GGCACCAA-3' (forward) and 5'-CTCTTTGATAT CACGCACGAT-3' (reverse), $63^{\circ} \mathrm{C}, 542 \mathrm{bp}$; NGF, 5'-GGCAAGTCAGCCTCTTGTAG-3' (forward) and 5'-GGCAAGTCAGCCTCTTCTTGTAGCCTT CC-3' (reverse), $60^{\circ} \mathrm{C}, 376 \mathrm{bp}$; BDNF, forward primer, 5'-CCCAGGGCAGGTTCGAGAGG-3' (forward) and 5'-CCGCCAGACATGTCCACTG-3' (reverse), $61^{\circ} \mathrm{C}, 350 \mathrm{bp}$; NT-3, 5'-TTACCAGAGCACCCTG CCCAAA-3' (forward) and 5'-ACCTGGTGTCCCC GAATGTCAA-3' (reverse), $61^{\circ} \mathrm{C}, 348 \mathrm{bp}$. After amplification, an aliquot of the PCR products was sub- jected to $2 \%$ agarose gel electrophoresis and visualized by ethidium bromide staining. The images of the bands were captured on FLA-5100 (Fujifilm, Tokyo, Japan). The optical density of each band was quantified by utilizing image-analysis software (Image J; National Institutes of Health). We carefully checked the reliability of the RT-PCR used in this study. That is, the quantification was performed within the range of linearity between the amount of RNA added and the obtained intensity of the band of a particular PCR product.

Western immunoblot analysis. Experimental procedures used were described previously (15). Briefly, the hippocampi were isolated and homogenized in $1 \mathrm{~mL}$ of lysis buffer containing $20 \mathrm{mM}$ Tris- $\mathrm{HCl}$ (pH 7.4), $150 \mathrm{mM} \mathrm{NaCl}, 2 \mathrm{mM}$ EDTA, 1\% NP-40, $10 \mu \mathrm{g} / \mathrm{mL}$ aprotinin, $10 \mu \mathrm{g} / \mathrm{mL}$ leupeptin, $50 \mathrm{mM}$ $\mathrm{NaF}, 1 \mathrm{mM} \mathrm{Na} \mathrm{VO}_{4}, 1 \mathrm{mM}$ phenylmethylsulfonyl fluoride (PMSF), $0.1 \%$ sodium dodecyl sulfate (SDS), and 1\% Na deoxycholate. Each tissue homogenate was then centrifuged, and the protein concentration of the resulting supernatant was determined with a BCA Protein Assay Kit (Thermo Scientific, Rockford, IL, USA). Samples (lysates of the hippo- 
campus: $5 \mu$ g protein per lane) were electrophoresed in $10 \%$ SDS-polyacrylamide gels, and the proteins were transferred to a Fluorotrans W membrane (Pall Life Sciences, Ann Arbor, MI, USA). The membranes were blocked with 5\% skim milk (Morinaga Milk products, Tokyo, Japan) in Tris-bufferd saline (TBS) $/ 0.1 \%$ Tween 20 for $1 \mathrm{~h}$ and then incubated with primary antibody against ERK1/2 (1: 1000, Cell signaling) or phospho-ERK1/2 (1:1000, Cell Signaling) at $4{ }^{\circ} \mathrm{C}$ overnight. After having been washed with $\mathrm{TBS} / 0.1 \%$ Tween 20 , the membranes were incubated with alkaline phosphatase-conjugated anti-rabbit IgG secondary antibody ( $1: 1000$; Promega, Madison, WI, USA) for $3 \mathrm{~h}$ at room temperature. Finally, the specific protein bands were developed with nitro blue tetrazolium and 5-bromo4-chloro-3-indorylphosphate p-toluidine salt. The intensity of immunoreactive bands was analyzed by use of image-analysis software (NIH Image J).

\section{RESULTS}

\section{Protection against the stress-induced anxiety-like} symptom

We evaluated the anxiolytic activity of DAEE in both protective and therapeutic aspects. First, the experimental protocol used to estimate the protective activity is illustrated in Fig. 2A. Mice were divided into 10 groups $(\mathrm{n}=8 /$ group $)$. Five groups were exposed to the CMSs, and the remaining 5 groups were not so exposed. Mice of each group were administered vehicle, DAEE $(20,100$ or $500 \mu \mathrm{g} / \mathrm{kg})$ or fluvoxamine $(1000 \mu \mathrm{g} / \mathrm{kg})$ once a day. The animals were then given 5 min to explore the elevated plus-maze 1 day after the final drug administration. The time spent in the open arms was significantly shorter in the stress group than in the non-stress group when respective vehicle-treated groups were compared, indicating that the CMSs had induced an anxiety-like state (Fig. 3A). However, the significant difference between the stress group and non-stress group disappeared by administration of DAEE or fluvoxamine. Furthermore, among the stress groups, the groups treated with DAEE $(500 \mu \mathrm{g} / \mathrm{kg})$ or fluvoxamine $(1000 \mu \mathrm{g} / \mathrm{kg})$ spent a significantly longer time in the open arms than the vehicle-treated group (Fig. 3A). Although among the non-stress-loaded groups, the time spent in open arms of the DAEE- or fluvoxamine-treated group tended to be greater than that for the vehicletreated group, the difference was not significant. The finding that the frequency of entry into all arms was constant in all experimental groups irrespective of
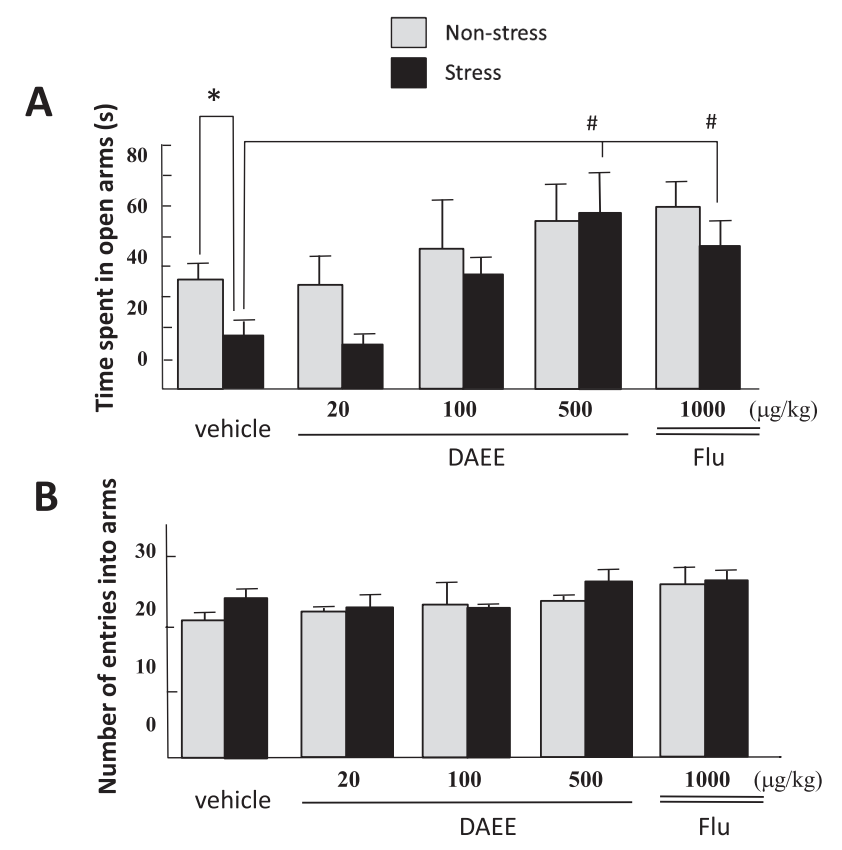

Fig. 3 Protective activity of DAEE against CMS-induced anxiety-like symptom. Mice were daily injected i.p. with vehicle, DAEE of various doses $(20,100$ or $500 \mu \mathrm{g} / \mathrm{kg})$ or fluvoxamine (Flu) at $1000 \mu \mathrm{g} / \mathrm{kg}$ for 3 weeks with or without exposure to the CMSs; and the time spent in open arms (A) and the number of entries into the arms $(B)$ in the elevated plus-maze test were then evaluated (see protocol $A$ in Fig. 2). A significant difference between the value of the stress group and that of the non-stress group was determined by Student's $t$-test as ${ }^{*} P<0.05$. Among the groups with CMS loading, significant differences from the value of the vehicle-treated mice were determined by one-way ANOVA with Tukey's test as ${ }^{\#} P<0.05$.

stress loading demonstrated that the locomotor activity was not influenced by the stress loading and/ or drug administration (Fig. 3B). These observations demonstrated that DAEE was as effective as fluvoxamine in protecting against the CMS-induced anxiety-like symptom.

Therapeutic effects on the stress-induced anxietylike symptom

The experimental protocol to estimate the therapeutic activity is shown in Fig. 2B. Mice were divided into 2 groups ( $n=40 /$ group). One group was exposed to the 3 types of CMSs for 21 days; and the other group, not. The anxiety-like symptom was evaluated by using the elevated plus-maze test 1 day after the completion of the CMS loading. The time spent in the open arms was significantly shorter in the stress group than that in the non-stress group without any change in the frequency of entry into all arms, demonstrating that the CMS induced the 


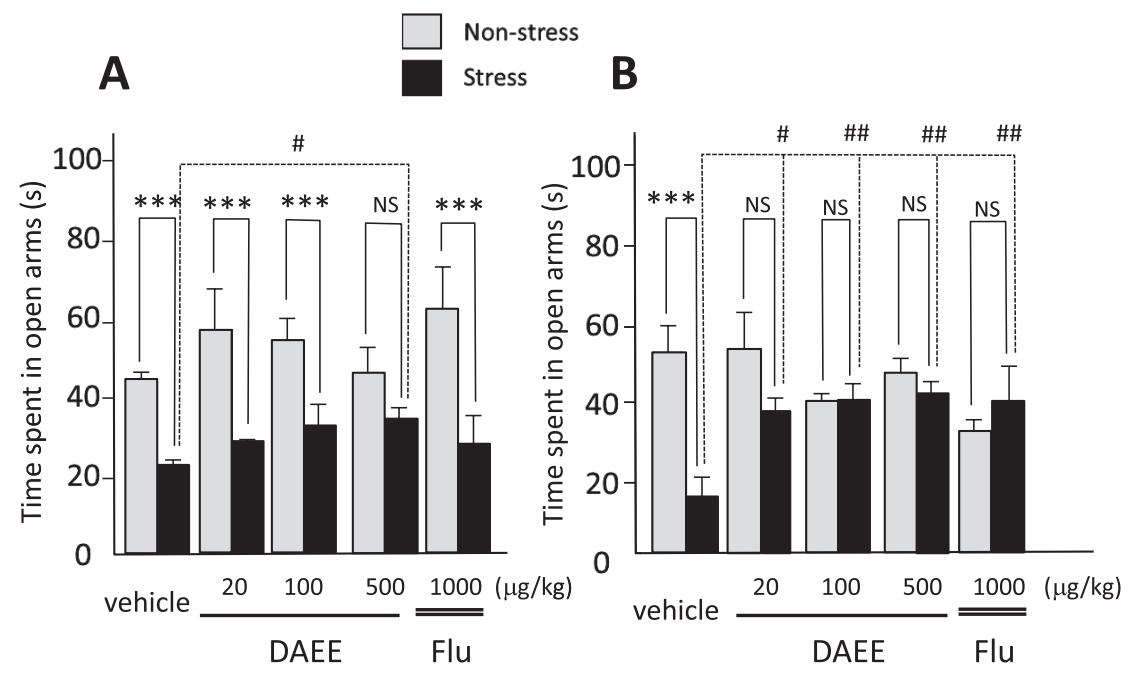

Fig. 4 Therapeutic activity of DAEE against CMS-induced anxiety-like symptom. Vehicle, DAEE at various doses (20, 100 or $500 \mu \mathrm{g} / \mathrm{kg}$ ) or fluvoxamine (Flu) at $1000 \mu \mathrm{g} / \mathrm{kg}$ was daily injected i.p. for 1 week (A) or 2 weeks (B) into mice that had been exposed or not to the CMSs (see protocol B in Fig. 2). Time(s) spent in open arms in the elevated plus-maze test was then evaluated. The significance of the difference between the value of the stress-exposed mice and that of the nonstress-exposed mice was determined by one-way ANOVA with Tukey's post hoc test as ${ }^{* * *} P<0.001$. NS means a lack of statistical significance. Furthermore, among the CMS-exposed groups, a significant difference from the value of the vehicletreated CMS-exposed mice was similarly determined by one-way ANOVA with Tukey's post hoc test as ${ }^{\#} P<0.05$, ${ }^{\# \#} P<0.01$.

anxiety-like symptom. Then, the stress group was divided into 5 groups (8/group), ensuring a similar behavioral trait distribution in each group. The nonstress group was also divided into 5 groups according to the same concept. Each group was then administered vehicle, DAEE $(20,100$ or $500 \mu \mathrm{g} / \mathrm{kg})$ or fluvoxamine $(1000 \mu \mathrm{g} / \mathrm{kg})$ once a day for 1 or 2 weeks, and given $5 \mathrm{~min}$ to explore the elevated plusmaze 1 day after the 7- or 14-day drug administration.

After 1-week treatment with vehicle, 20 or $100 \mu \mathrm{g} /$ $\mathrm{kg}$ of DAEE or $1000 \mu \mathrm{g} / \mathrm{kg}$ of fluvoxamine, the time spent in the open arms was still significantly shorter in the stress groups than in the non-stress ones; however, after the 1-week treatment with $500 \mu \mathrm{g} / \mathrm{kg}$ of DAEE, this difference became non-significant (Fig. 4A). On the other hand, after the 2-week treatment, all groups except for the vehicle-treated group lost statistical significance in the time spent in the open arms between stress and non-stress groups (Fig. 4B). As the frequency of entry into all arms remained unchanged among all groups after 1- or 2-week treatment (data not shown), the locomotor activity was not influenced by the stress and/or drug administration. These observations demonstrate that DAEE had an anxiolytic-like activity similar to that of fluvoxamine, a currently used SSRI. Furthermore, although more detailed experiments are necessary, it may be plausible that DAEE ameliorated the stress- induced anxiety more rapidly than fluvoxamine.

Influence of DAEE on the neurotrophin $m R N A$ level in the hippocampus

After exposure to the CMSs for 20 days, the mRNA level of BDNF or NT-3, but not that of NGF, was substantially and significantly reduced in the hippocampus (Fig. 5). However, the reduction in the expression of BDNF or NT-3 mRNA was attenuated by the administration of DAEE once a day for 21 days during stress-loading period according to the experimental protocol A (see Fig. 1A) (Fig. 5). The expression level of NGF mRNA, which was not influenced by the stress, was significantly enhanced by the administration with $500 \mu \mathrm{g} / \mathrm{kg}$ of DAEE. The effect of DAEE was not dose-dependent, although reason was unknown.

Influence of DAEE on the stress-induced decrease in the level of phosphorylated ERK1/2 in the hippocampus

Effects of the CMS exposure and/or DAEE administration to the mice on the level of the phosphorylated ERK1/2 (pERK1/2) in the hippocampus were investigated by using the 2 different experimental protocols (see Fig. 2). First, mice were exposed to the CMSs for 20 days with a daily administration of vehicle or various doses of DAEE according to the experimental protocol A. This experiment was aimed 


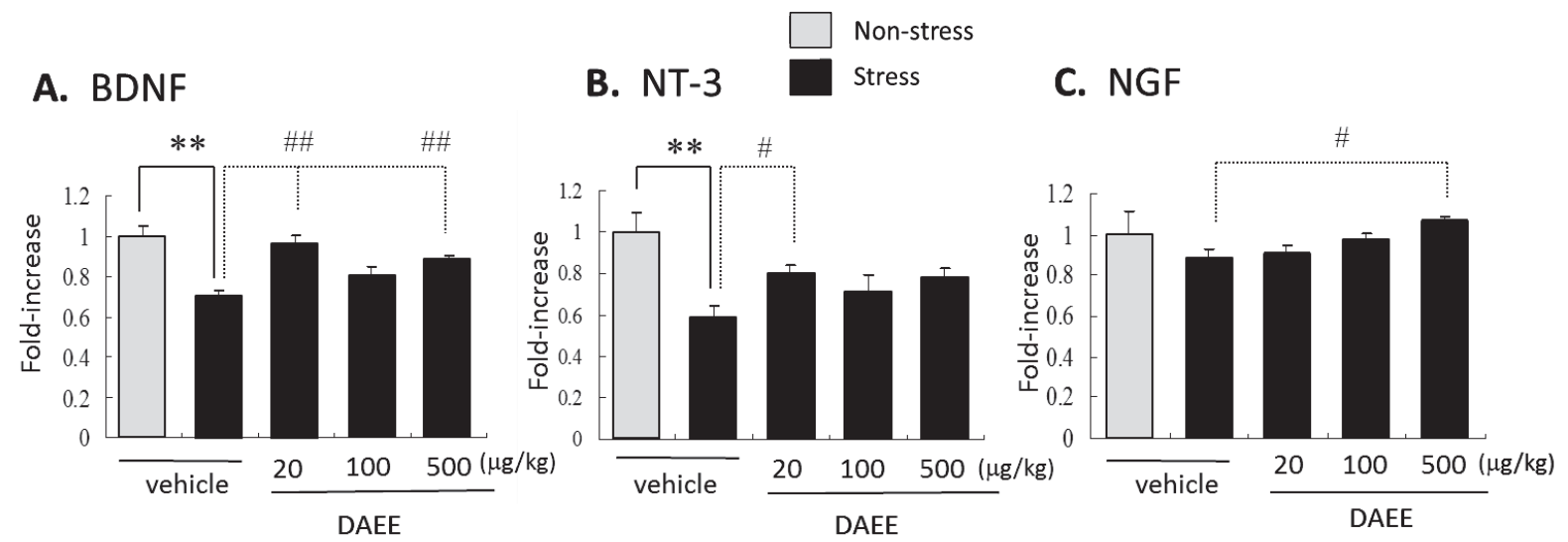

Fig. 5 Ameliorative activity of DAEE toward CMS-induced mRNA expression in the hippocampus. After a daily i.p. injection of vehicle or DAEE at various doses for 20 days during CMS-loading (see protocol A in Fig. 2), the hippocampus was dissected out 1 day after the final DAEE administration and subjected to RT-PCR analysis. The intensity of the target DNA band was normalized by the intensity of the $\beta$-actin DNA band, and the values were expressed as fold-increase over the value for the vehicle-treated group without stress exposure taken as "1." Significant differences from the value of the nonstress group were determined by one-way ANOVA with Tukey's test as ${ }^{*} P<0.05,{ }^{*} P<0.01$. Among CMS-exposed groups, significant differences from the value of the vehicle-treated group were also similarly determined as ${ }^{\#} P<0.05$, ${ }^{\# \#} P<0.01$.

at evaluating whether DAEE acted protectively against the stress-induced reduction in the pERK1/2 level. The results are shown in Fig. 6A. The ratio of the intensity of the band of pERK $1 / 2$ to that of total ERK1/2 was expressed as fold-increase over the value (taken as "1") for the vehicle-treated group without stress, and the value was reduced by the stress. However, such stress-induced reduction in the ratio was attenuated by the treatment with $500 \mu \mathrm{g}$ / $\mathrm{kg}$ of DAEE (Fig. 6A).

Second, mice were exposed to the CMSs for 20 days, and given the vehicle or various doses of DAEE from 1 day after the end of the CMSs for 2 weeks according to the protocol $\mathrm{B}$. The hippocampi were dissected out 1 day after the end of the final administration, $i$. e., 15 days after the end of the CMSs. The ratio of the intensity of the band of pERK $1 / 2$ to that of total ERK $1 / 2$ in the CMS groups was still significantly lower than that in the non-stress group, and this reduction was attenuated by the treatment with 100 or $500 \mu \mathrm{g} / \mathrm{kg}$ of DAEE (Fig. 6B). These observations demonstrated that the anxiety-like symptom was inversely proportional to the level of pERK1/2 in the hippocampus and that both were coincidently regulated by DAEE, suggesting the involvement of hippocampal ERK1/2 signaling in the expression of the anxiety-like state.

\section{DISCUSSION}

Neurotrophins are a family of neurotrophic factor proteins that include NGF, BDNF, and NT-3. Each neurotrophin binds to a specific Trk receptor tyrosine kinase (6), which binding causes autophosphorylation of the receptor to trigger signal transduction cascades of pathways involving MAPK/ERK1/2, phosphatidylinositol 3-kinase, and phospholipase $\mathrm{C}-\gamma$ (20). These signals regulate various genes involved in neuronal survival and maintenance of neuronal functions including synaptic plasticity in adulthood $(16,28)$. These activities have been expected to be therapeutically useful for particular neurological disorders. However, the clinical trials of neurotrophins for some neurological disorders have not been successful (4), probably because many technical and pharmacological issues such as instability of the proteins and/or a lack of appropriate delivery systems are problematic. To overcome these issues, we recently developed trans-2-decenoic acid derivatives as stable and small molecules with neurotrophin-like activity (29). Several results using animal models indicate that BDNF infusion into the brain produces an antidepressant effect $(9,31)$. Therefore, in an earlier study we examined the effects of DAEE, a 2-decenoic acid derivative with the most potent activity in vitro, on a stress-induced depression model in mice. Our results demonstrated that DAEE protected against the depression when administered i.p. simultaneously with the stress loading, and ameliorated the stress-induced depression (12), suggesting that DAEE might mimic BDNF actions toward anxiety symptoms.

Therefore, in the present study we examined the effects of DAEE on a stress-induced anxiety-like 

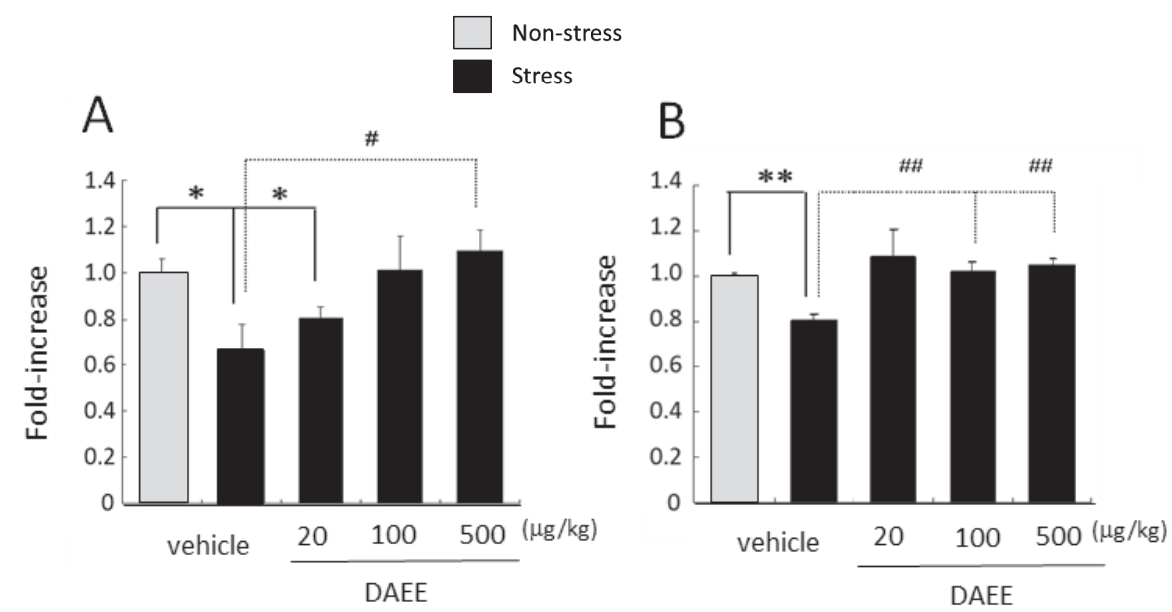

Fig. 6 Ameliorative activity of DAEE against the CMS-induced decrease in the hippocampal pERK1/2 level. A: After daily i.p. injection of vehicle or DAEE at various doses to the mice for 21 days during CMS exposure (see protocol A in Fig. 2), the hippocampi were dissected out 1 day after the final DAEE administration. B: Mice were injected with vehicle or DAEE at various doses for 2 weeks after the end of the CMS exposure (see protocol B in Fig. 2), and the hippocampi were dissected out 1 day after the end of the DAEE administration. The ratio of the intensity of the pERK $1 / 2$ band to that of the total ERK1/2 band was calculated by Western immunoblotting, and the values were expressed as fold-increase over the value of the vehicle-treated group without stress exposure taken as "1." The significance of differences between the values of the stress-exposed and the non-stress-exposed mice was determined by one-way ANOVA with Tukey's post hoc test as ${ }^{*} P<0.05$, ${ }^{* *} P<0.001$. For differences between values of CMS-exposed groups, the significance was similarly determined by the same statistical treatment as ${ }^{\#} P<0.05,{ }^{\# \#} P<0.01$.

model in mice. The results demonstrated that DAEE protected against the anxiety-like symptom expected to be induced by the stress-loading (Fig. 3), and ameliorated the symptom that had been evoked (Fig. 4). These advantageous activities were accompanied by restoration to normal of the stress-induced reduction in the expression of BDNF and NT-3 mRNAs (Fig. 5) or in the level of pERK1/2 (Fig. 6) in the hippocampus. Previously we reported that, in neurons cultured from the mouse cerebral cortex/ hippocampus, DAEE activates CREB predominantly through ERK1/2 activation, not through other pathways such as $\mathrm{cAMP} /$ protein kinase $\mathrm{A}$, and that it enhances the expression of BDNF and NT-3 mRNAs, but not that of NGF mRNA, and increases the contents of synapse-specific proteins such as synaptophysin, synapsin-1, and syntaxin (29). These observations suggest that facilitation of neurotrophin-like signal transduction in the hippocampus may be one of the mechanisms underlying the anxiolytic-like activities of DAEE.

DAEE significantly reversed the stress-induced decrease in BDNF mRNA expression in the hippocampus (Fig. 5), which action may be comparable to that of antidepressants that work via the activation of a specific set of genes including the BDNF gene (1). Furthermore, DAEE also restored to normal the stress-dependent reduction in the pERK1/2 level
(Fig. 6). It should be discussed whether or not DAEE activates ERK1/2 directly or indirectly via DAEEinduced BDNF. However, it is unlikely that DAEEinduced BDNF predominantly activated ERK1/2 because of our previous observation showing that ERK $1 / 2$ in cultured neurons is phosphorylated as early as $15 \mathrm{~min}$ after exposure to DAEE (29). pERK $1 / 2$ thus activated would mediate downstream of neurotrophin-like signaling to lead to neurogenesis in the hippocampus. Therefore, DAEE might activate ERK1/2 more rapidly than currently used antidepressants, because these antidepressant drugs are thought to activate the ERK1/2/CREB signaling pathway via antidepressant-induced $\operatorname{BDNF}(5,8)$.

BDNF gene expression and activation of its highaffinity receptor, TrkB, are necessary in the amygdala, hippocampus, and prefrontal cortex for the formation of fear memories (3). Therefore, it is important to examine the effects of DAEE on the amygdala for understanding its actions against the anxiety-like symptom. Furthermore, it is critically important to clarify the molecular mechanisms of DAEE especially prior to activation of MAPK/ERK kinases. For example, 7,8-dihydroxyflavone was identified as a potential small-molecule agonist of the TrkB receptor (14). When systemically administered to mice, this flavone provokes TrkB receptor dimerization and autophosphorylation, resulting in 
the activation of downstream signaling (14). A single systemic dose of this flavone activates TrkB receptors within the amygdala and enhances both fear acquisition and extinction in naïve mice (2). However, our preliminary results indicated that DAEE did not activate TrkB, suggesting some mechanism different from that of 7,8-dihydroxyflavone. More investigation of 2-decenoic acid derivatives including DAEE is necessary to understand their action mechanism and to assess their potential for medical use.

Our present results demonstrate that DAEE and probably its related compounds are effective in ameliorating the observed stress-inducible symptom of anxiety and suggest that they may become a promising tool as a new anxiolytic.

\section{ACKNOWLEDGEMENTS}

This work was supported in part by a grant from the program Grants-in-Aid for Scientific Research (B) of the Japan Society for the Promotion of Science.

\section{REFERENCES}

1. Anacker C, Zunszain PA, Cattaneo A, Carvalho LA, Garabedian MJ, Thuret S, Price J and Pariante CM (2011) Antidepressants increase human hippocampal neurogenesis by activating the glucocorticoid receptor. Mol Psychiatry 16, 738-750.

2. Andero R, Heldt SA, Ye K, Liu X, Armario A and Ressler KJ (2011) Effect of 7,8-dihydroxyflavone, a small-molecule TrkB agonist, on emotional learning. Am J Psychiatry 168, $163-172$.

3. Andero R and Ressler KJ (2012) Fear extinction and BDNF: translating animal models of PTSD to the clinic. Genes Brain Behav 11, 503-512.

4. Apfel SC (2001) Neurotrophic factor therapy-prospects and problems. Clin Chem Lab Med 39, 351-355.

5. Baj G, D'Alessandro V, Musazzi L, Mallei A, Sartori CR, Sciancalepore M, Tardito D, Langone F, Popoli M and Tongiorgi E (2012) Physical exercise and antidepressants enhance BDNF targeting in hippocampal CA3 dendrites: further evidence of a spatial code for BDNF splice variants. Neuropsychopharmacology 37, 1600-1611.

6. Barbacid M (1995) Structural and functional properties of the TRK family of neurotrophin receptors. Ann NY Acad Sci 766, 442-458.

7. Barde YA (1994) Neurotrophins: a family of proteins supporting the survival of neurons. Prog Clin Biol Res 390, 4556.

8. Cottingham C, Jones A and Wang Q (2012) Desipramine selectively potentiates norepinephrine-elicited ERK1/2 activation through the $\alpha 2 \mathrm{~A}$ adrenergic receptor. Biochem Biophys Res Commun 420, 161-165.

9. Duman RS (2002) Synaptic plasticity and mood disorders. Mol Psychiatry 7, S29-34.

10. Duman RS (2004) Role of neurotrophic factors in the etiology and treatment of mood disorders. Neuromolecular Med $\mathbf{5}$, $11-25$.
11. Duman RS and Monteggia LM (2006) A neurotrophic model for stress-related mood disorders. Biol Psychiatry 59, 11161127.

12. Furukawa S (2009) Development of therapeutic drugs for depression and Alzheimer's disease. Chemical Engineering (in Japanese) 54, 18-24.

13. Gould E and Tanapat P (1999) Stress and hippocampal neurogenesis. Biol Psychiatry 46, 1472-1479.

14. Gould E, Tanapat P, McEwen BS, Flugge G and Fuchs E (1998) Proliferation of granule cell precursors in the dentate gyrus of adult monkeys is diminished by stress. Proc Natl Acad Sci USA 95, 3168-3171.

15. Hirakawa A, Shimizu K, Fukumitsu H, Soumiya H, Iinuma $\mathrm{M}$ and Furukawa S (2010) 2-Decenoic acid ethyl ester, a derivative of unsaturated medium-chain fatty acids, facilitates functional recovery of locomotor activity after spinal cord injury. Neuroscience 171, 1377-1385.

16. Huang EJ and Reichardt LF (2001) Neurotrophins: roles in neuronal development and function. Annu Rev Neurosci 24, $677-736$.

17. Ito $\mathrm{N}$, Nagai $\mathrm{T}$, Yabe $\mathrm{T}$, Nunome $\mathrm{S}$, Hanawa $\mathrm{T}$ and Yamada H (2006) Antidepressant-like activity of a Kampo (Japanese herbal) medicine, Koso-san (Xiang-Su-San), and its mode of action via the hypothalamic-pituitary-adrenal axis. Phytomedicine 13, 658-667.

18. Jang SW, Liu X, Chan CB, France SA, Sayeed I, Tang W, Lin X, Xiao G, Andero R, Chang Q, Ressler KJ and Ye K (2010) Deoxygedunin, a natural product with potent neurotrophic activity in mice. PLoS One 5, e11528.

19. Jang SW, Liu X, Yepes M, Shepherd KR, Miller GW, Liu Y, Wilson WD, Xiao G, Blanchi B, Sun YE and Ye K (2010) A selective TrkB agonist with potent neurotrophic activities by 7,8-dihydroxyflavone. Proc Natl Acad Sci USA 107, 26872692.

20. Kaplan DR and Miller FD (2000) Neurotrophin signal transduction in the nervous system. Curr Opin Neurobiol 10, 381-391.

21. Karpova NN, Pickenhagen A, Lindholm J, Tiraboschi E, Kulesskaya N, Agústsdóttir A, Antila H, Popova D, Akamine Y, Bahi A, Sullivan R, Hen R, Drew LJ and Castrén E (2011) Fear erasure in mice requires synergy between antidepressant drugs and extinction training. Science 334, 1731-1734.

22. Kasai M, Fukumitsu H, Soumiya H and Furukawa S (2010) Ethanol extract of chinese propolis facilitates functional recovery of locomotor activity after spinal cord injury. Evid Based Complement Alternat Med 2011. pii. 749627. Epub. 2010, Sep. 8.

23. Kirtley A and Thomas KL (2010) The exclusive induction of extinction is gated by BDNF. Learn Mem 17, 612-619.

24. Kitayama N, Vaccarino V, Kutner M, Weiss P and Bremner JD (2005) Magnetic resonance imaging (MRI) measurement of hippocampal volume in posttraumatic stress disorder: a meta-analysis. J Affect Disord 88, 79-86.

25. Lang UE, Hellweg R, Kalus P, Bajbouj M, Lenzen KP, Sander T, Kunz D and Gallinat J (2005) Association of a functional BDNF polymorphism and anxiety-related personality traits. Psychopharmacology (Berl) 180, 95-99.

26. Lattal KM, Barrett RM and Wood MA (2007) Systemic or intrahippocampal delivery of histone deacetylase inhibitors facilitates fear extinction. Behav Neurosci 121, 1125-1131.

27. Lo DC (1995) Neurotrophic factors and synaptic plasticity. Neuron 15, 979-981.

28. Lu B (2003) BDNF and activity-dependent synaptic modulation. Learn Mem 10, 86-98. 
29. Makino A, Inuma M, Fukumitsu H, Soumiya H, Furukawa $\mathrm{Y}$ and Furukawa S (2010) 2-Decenoic acid ethyl ester possesses neurotrophin-like activities to facilitate intracellular signals and increase synapse-specific proteins in neurons cultured from embryonic rat brain. Biomed Res 31, 379-386.

30. Sheline YI, Gado MH and Kraemer HC (2003) Untreated depression and hippocampal volume loss. Am J Psychiatry 160, 1516-1518.

31. Siuciak JA, Lewis DR, Wiegand SJ and Lindsay RM (1997) Antidepressant-like effect of brain-derived neurotrophic fac- tor (BDNF). Pharmacol Biochem Behav 56, 131-137.

32. Ueyama T, Kawai Y, Nemoto K, Sekimoto M, Tone S and Senba E (1997) Immobilization stress reduced the expression of neurotrophins and their receptors in the rat brain. Neurosci Res 28, 103-110.

33. Vale AL, Green S, Montgomery AM and Shafi S (1998) The nitric oxide synthesis inhibitor L-NAME produces anxiogenic-like effects in the rat elevated plus-maze test, but not in the social interaction test. J Psychopharmacol 12, 268-272. 